\title{
A utilização da noção de relação ao saber (rapport au savoir) no contexto do Ensino de Matemática: mapeamento inicial de referências bibliográficas*
}

\author{
The use of the notion of relation to knowledge \\ (rapport au savoir) in the context of Mathematics Teaching: \\ Mapping in bibliographic references
}

José Dilson Beserra Cavalcanti ${ }^{1}$ • http://orcid.org/0000-0002-6125-3867 Anna Paula Avelar Brito Lima² . https:// orcid.org/0000-0003-1471-228X

\begin{abstract}
Resumo: Esse artigo apresenta um esboço do cenário da produção científica acerca da noção de relação ao saber no contexto específico do Ensino de Matemática. A natureza do estudo foi exploratória e a metodologia utilizada foi o mapeamento em pesquisa educacional. De um total de 241 referências bibliográficas repertoriadas no mapeamento geral, 68 referências estão vinculadas ao contexto do Ensino de Matemática, o que corresponde a 28\%. Como resultado principal, destacamos um mapa inicial com as descrições de quem utilizou a noção em teses e dissertações; quem foram os orientadores e quais foram os programas de pós-graduação nos quais estas produções foram desenvolvidas; quem foram os autores que publicaram artigos científicos e em quais periódicos. Acreditamos, por fim, que tais informações podem ser utilizadas como coordenadas relevantes para aqueles que pretendem estudar e utilizar a noção de relação ao saber em suas investigações no contexto do Ensino de Matemática.
\end{abstract}

Palavras-chave: Ensino de matemática. Produção científica. Mapeamento. Levantamento bibliográfico.

\begin{abstract}
This paper presents an initial sketch of the scientific production scenario about the notion of relation to the knowledge in the particular context of Mathematics Teaching. The nature of the study was exploratory, and the methodology used was the mapping in educational research. In the general mapping, 241 bibliographical references were cataloged. Of this total, 68 references are linked to the context of Mathematics Teaching, which corresponds to $28 \%$. As a main result, we highlight an initial map with the descriptions of those who used the notion in thesis and dissertations, who were the advisors and the graduate programs in which these productions were developed, who were the authors who published scientific articles and in which journals. We believe, finally, that such information can be used as relevant coordinates to those who intend to study and use the notion of relation to knowledge in their investigations in the context of Mathematics Teaching.
\end{abstract}

Keywords: Mathematics teaching. Scientific production. Mapping. Bibliographic research.

\footnotetext{
${ }^{*}$ Esse trabalho apresenta parte dos resultados obtidos na tese de doutorado do primeiro autor.

${ }^{1}$ Universidade Federal de Pernambuco (UFPE), Centro Acadêmico do Agreste, Programa de Pós-Graduação

Educação em Ciências e Matemática, Caruaru, PE, Brasil. Email: <dilsoncavalcanti@gmail.com>.

${ }^{2}$ Universidade Federal Rural de Pernambuco (UFRPE), Programa de Pós-Graduação em Ensino de Ciências e

Matemática, Recife, PE, Brasil.
} 


\section{A noção de relação ao saber: considerações gerais}

A noção de relação ao saber surge na literatura científica francesa a partir da segunda metade do século XX. No que diz respeito à história dessa noção, em Cavalcanti (2015) foram analisados diversos trabalhos que abordam esse tema, especialmente Beillerot (1989), e propomos considerar cinco fases.

A primeira delas corresponde ao surgimento da expressão rapport au savoir na literatura científica nos campos da Psicanálise - com Jacques Lacan, em 1960, no texto Subversion du sujet et dialectique du désir, conferência proferida no Colloque Philosophique International (LACAN, 1966) - e da Sociologia - com Pierre Bourdieu e Jean-Claude Passeron na obra La Reprodution (BOURDIEU; PASSERON, 1970).

A segunda fase pode ser compreendida como movimentos de propagação da expressão. Essa propagação se deu em dois contextos. O primeiro corresponde à utilização da expressão nos campos em que ela surgiu, isto é, na Psicanálise e na Sociologia. Sendo assim, no campo psicanalítico, a expressão foi empregada, ainda na década de 1960, por pesquisadores de orientação lacaniana, destacando-se Piera Aulagnier (AULAGNIER, 2009) que a utilizou em um texto ${ }^{3}$ abordando a problemática psicanalítica do desejo. No campo da Sociologia, conforme Beillerot (1989), a expressão rapport au savoir teria sido utilizada na década de 1970 nos trabalhos de pesquisadores de inclinação marxista, tais como Marcel Lesne e Bernard Charlot, sendo importante ressaltar que esse último desenvolve, ainda nessa época, a ideia de relação social ao saber. O segundo contexto é respectivo à importação da expressão por outros campos, especificamente, da Formação de Adultos (CAVALCANTI, 2015; LAOT, 2009) e da Didática ${ }^{4}$.

A terceira fase seria referente à institucionalização da relação ao saber como noção teórica. O processo de institucionalização se dá no final da década de 1980 e, principalmente, na década de 1990, considerando-se seu reconhecimento como tal e as diferentes abordagens teóricas que são desenvolvidas. Nessa fase, em Cavalcanti (2015) propõe-se que o núcleo duro epistemológico ${ }^{5}$ da noção compreenda três perspectivas teóricas, comumente mencionadas como abordagens da relação ao saber.

- A abordagem identificada como microssociológica/sociológica desenvolvida por Bernard Charlot e sua equipe de pesquisa Education, Socialisation et Collectivités Locales (ESCOL) e a abordagem socio-antropológica desenvolvida por Bernard Charlot;

\footnotetext{
${ }^{3}$ AULAGNIER-SPAIRANI, P. Le désir de savoir dans ses rapports à la transgression. L'Inconscient, n. 1, p. $109-125,1967$.

${ }^{4}$ Charlot (2000) aponta que a expressão rapport au savoir também foi utilizada na década de 1970 em textos de André Giordan, pesquisador em Didática das Ciências. Abordamos a questão da utilização da expressão rapport au savoir no campo das Didáticas em Cavalcanti (2015).

${ }^{5}$ Reconhecemos que a inspiração da expressão núcleo duro epistemológico provém dos trabalhos de autoria de Lakatos. No entanto, esclarecemos que o uso que fizemos dessa expressão foi contextual para se referir ao conjunto de teorizações acerca da relação ao saber na fase de institucionalização da noção. Dessa maneira, justificamos que esse uso não implica, necessariamente, em um estudo nos moldes do programa de pesquisa "lakatosiano".
} 
- A abordagem identificada como clínica/socio-clínica/psicanalítica desenvolvida por Jacky Beillerot e sua equipe de pesquisa Savoir et Rapport au Savoir do Centre de Recherche Éducation et Formation (CREF) da Université Paris X em Nanterre;

- A abordagem desenvolvida por Yves Chevallard.

As duas primeiras abordagens estão relacionadas ao desenvolvimento da noção no campo das Ciências da Educação, embora conservando bases teóricas sociológicas e psicanalíticas, respectivamente. Desse modo, Bernard Charlot e a equipe ESCOL desenvolvem, num primeiro momento, uma abordagem com base na Sociologia, que comumente é mencionada como abordagem sociológica ou microssociológica (BAUTIER; CHARLOT; ROCHEX, 1992). Essa abordagem é fundada a partir de uma reflexão crítica e investigações sobre a problemática do fracasso escolar. Em outra ocasião, no contexto de elaboração de uma sociologia do sujeito, Charlot (2000) promove uma reflexão mais ampla privilegiando um ponto de vista antropológico (optamos por distinguir como socioantropológica).

Já Jacky Beillerot e a equipe do CREF desenvolvem uma abordagem comumente mencionada como clínica (BEILLEROT; BLANCHARD-LAVILLE; MOSCONI, 1996), socioclínica (MOSCONI, 2008) e/ou psicanalítica (HATCHUEL, 2005). Essa abordagem pode ser situada como um prolongamento do estudo realizado por Jacky Beillerot (BEILLEROT, 1987) em sua tese de doutorado. Nessa ocasião, o autor defende que a relação ao saber envolve uma dimensão social e uma dimensão psíquica.

Nas abordagens de bases psicanalíticas, com Jacky Beillerot e equipe Savoir et Rapport an Savoir, e sociológicas, com Bernard Charlot e equipe ESCOL, a relação ao saber é centralizada a partir do sujeito, como parte constitutiva dele. Sendo assim, representa uma relação, indissociavelmente, singular e social (CHARLOT, 2000), podendo ser entendida como uma disposição íntima constituída num quadro de gramática social (BEILLEROT, 1989). Nessa perspectiva, é considerada uma noção de contornos teóricos amplos.

Diferentemente dessas duas abordagens, a teorização elaborada por Yves Chevallard (2003, 2015) não considera bases teóricas psicanalíticas nem sociológicas. Sendo assim, o campo no qual é desenvolvida é o da Didática da Matemática no quadro de sua Teoria Antropológica do Didático (TAD). Portanto, essa teorização da noção de relação ao saber é reconhecida como abordagem antropológica (CHEVALLARD, 2003, 2015). No entanto, para não confundir essa abordagem com a desenvolvida por Charlot (2000), propomos identifica-la como didático-antropológica.

Esclarecemos que a teorização desenvolvida por Chevallard $(2003,2015)$ centraliza a noção a partir do saber, colocando em evidência o quadro das relações pessoal e institucional ao saber. Sua operacionalização, assim, é diferente das abordagens de bases psicanalíticas e/ou sociológicas. Uma das questões centrais dessa diferença, conforme Cavalcanti (2015), pode ser o fato da rigidez teórica com a qual a noção é construída e definida por Chevallard no seio da TAD.

Como podemos ver, mesmo que superficialmente, o contexto da institucionalização da relação ao saber, como noção, aponta que sua natureza teórica é multidisciplinar, complexa e multirreferencial. Tais características podem ser evidenciadas na quarta fase de desenvolvimento da noção, que corresponde ao movimento de difusão no cenário francófono. Conforme Cavalcanti (2015), a relação ao saber é reconhecida nesse cenário como uma das principais noções-chave para o estudo e a pesquisa nos campos da Educação e Formação, bem como das Didáticas dos Conteúdos Específicos. 
É interessante ressaltar que é possível observar que as abordagens clínica/socioclíni$\mathrm{ca} /$ psicanalítica e microssociológica/sociológica/socioantropológica figuram bem nos diferentes contextos de pesquisa dos campos da Educação, Formação e Didáticas dos Conteúdos Específicos. Já a abordagem didático-antropológica, de Yves Chevallard, é particularmente situada no campo Didática.

Considerando a difusão da noção para além do cenário francófono, Cavalcanti (2015) descreve a quinta fase de desenvolvimento da noção de relação ao saber como movimento de universalização. Nessa perspectiva, argumenta que, no Brasil, a noção também é bastante utilizada em diversos contextos do campo da Educação (no geral) e do Ensino, especialmente, de Ciências e Matemática. Além disso, considera que a relação ao saber pode ser, no contexto da literatura científica brasileira, uma das noções-chave para o estudo e pesquisa de problemáticas concernentes a esses campos.

Nesse sentido, Cavalcanti (2015) realizou dois mapeamentos em referências bibliográficas acerca da utilização da noção de relação ao saber na literatura científica brasileira. No primeiro mapeamento, inventariou todas as referências bibliográficas (respectivas às teses, dissertações, artigos publicados em periódicos e em anais de eventos) que identificou com alguma menção no título à noção de relação ao saber, realizando um mapeamento geral na literatura científica brasileira. No segundo mapeamento, por sua vez, focou nas referências bibliográficas vinculadas ao contexto particular do campo do Ensino de Matemática ${ }^{6}$.

Desse modo, a finalidade do presente artigo é apresentar os resultados obtidos a partir desse segundo mapeamento. Consideramos que esse mapeamento pode ser compreendido como uma cartografia inicial da utilização da noção de relação ao saber na literatura científica respectiva ao campo do Ensino de Matemática.

\section{Metodologia: mapeamento em referências bibliográficas}

A produção do conhecimento científico é um processo bastante dinâmico e complexo que envolve muitos fatores como, por exemplo, formação dos pesquisadores, fomento, interlocução e inserção em um campo teórico. O desenvolvimento da produção em determinado campo ou sobre determinada temática constitui algo como uma rede ou programa de pesquisa. Nesse contexto, cada trabalho (e.g. tese, dissertação, artigo, comunicação) desenvolvido é uma parte dessa rede ou programa.

\footnotetext{
${ }^{6}$ Reconhecemos que às vezes, na literatura científica, os termos 'Ensino de Matemática', 'Educação Matemática' e 'Didática da Matemática' são utilizados como sinônimos ou sem uma devida distinção. Por essa razão, esclarecemos que em relação ao termo 'Educação Matemática', compreendemo-lo como referente a um campo científico e profissional com estatuto epistemológico próprio, no qual, entre outras temáticas, está incluída a do 'ensino de Matemática'. No entanto, a temática do 'ensino de Matemática' às vezes pode ser objeto de investigação de outros campos científicos sem, necessariamente, estar vinculada ao campo da 'Educação Matemática'. Em razão disso, optamos por utilizar o termo 'Ensino de Matemática' para designar um campo mais amplo no qual podem estar inseridos a temática do 'ensino de Matemática' enquanto objeto de investigação do campo da 'Educação Matemática', mas também de outros campos científicos. O termo 'Didática da Matemática' foi utilizado especificamente para se referir à disciplina científica francesa.
} 
Com o aumento cada vez maior da produção científica, diversas áreas do conhecimento têm buscado maneiras de compreender seu desenvolvimento e estado atual. Dessa maneira, a investigação e sistematização daquilo que se é produzido sobre determinada temática ou campo teórico é importante para identificar tendências, avaliar a situação do processo de desenvolvimento, sistematizar questões e sinalizar novas perspectivas.

Como já mencionamos anteriormente, em nosso processo inicial de pesquisa sobre a noção de relação ao saber, evidenciamos que havia uma grande quantidade de produção sobre essa temática na literatura científica brasileira. No entanto, não encontramos trabalhos específicos de levantamento bibliográfico ou estado da arte acerca das pesquisas desenvolvidas que nos permitissem vislumbrar o conjunto da produção bibliográfica nacional. Como parte de nossa tese, desenvolvemos um estudo exploratório sobre a produção científica brasileira acerca dessa noção, realizando um mapeamento geral e um específico, sendo o mapeamento específico restrito às referências bibliográficas correspondentes ao campo do Ensino de Matemática.

Um estudo dessa natureza poderia ser realizado a partir de diferentes enfoques metodológicos. Nossa opção foi o mapeamento em pesquisa educacional desenvolvido por Salett Biembengut (BIEMBENGUT, 2003, 2008). Alinhamo-nos com Biembengut (2008, p. 71) quando assinala que "cada pesquisa que se desencadeia insere-se em uma rede preexistente e seu valor é relativo à contribuição a essa rede".

Concordamos com a autora quando ela chama a atenção para o fato de que muitos dos trabalhos não têm partido das últimas pesquisas nem "apresenta o que já existe sobre o tema, quantos, quem e onde já fizeram algo a respeito, que avanços foram conseguidos e quais problemas estão em aberto para serem levados adiante” (BIENBENGUT, 2008, p. 73, grifos do autor).

A partir das ideias e dos respectivos questionamentos desenvolvidos por Biembengut (2008), Cavalcanti (2015) se propõe a realizar uma distinção sobre o direcionamento do mapeamento. Assim, os questionamentos “quantos, quem e onde já fizeram algo a respeito?” apontariam para um estudo exploratório horizontal se concentrando mais no relevo observável das produções científicas, isto é, na topologia dos territórios. Já os questionamentos “que avanços foram conseguidos e quais problemas estão em aberto para serem levados adiante" indicariam um estudo vertical que poderia ter como orientação o que está sob (isto é, os trabalhos já desenvolvidos - indicariam tendências) e o que está sobre (isto é, os trabalhos que podem ser desenvolvidos - indicariam perspectivas) a superfície da literatura científica.

Nosso mapeamento está relacionado ao primeiro caso - quantos, quem e onde já fizeram algo a respeito. Para melhor investigarmos, delimitamos quatro territórios exploratórios da produção científica: as teses, dissertações, artigos em periódicos e em anais de eventos ${ }^{7}$.

Sendo assim, buscamos investigar: quantas teses, dissertações, artigos em periódicos e anais de eventos? Quem foram os autores e orientadores ${ }^{8}$ das teses, dissertações, artigos em

\footnotetext{
${ }^{7}$ Em nossa exploração no território eventos, deparamo-nos com uma grande quantidade de referências bibliográficas, não sendo possível concluir a análise em razão do tempo limitado. Embora a análise ainda se encontre em processo, optamos por utilizar apenas informações do total de referências desse território para fins de sinalizar uma visão geral dos quatro territórios.

${ }^{8}$ No caso das teses e dissertações.
} 
periódicos e anais de eventos? Onde foram desenvolvidos/publicados, isto é, em quais programas de pós-graduação, periódicos, eventos. Além dessas variáveis, consideramos também a evolução da produção científica, organizando as informações por ano e, às vezes, agrupando por décadas.

Acreditamos que esse mapeamento, compreendido como horizontal, é fundamental para outros estudos, inclusive, para o desenvolvimento posterior de mapeamentos verticais (no sentido de identificar tendências e o estado atual e projetar perspectivas de pesquisas futuras).

A natureza de nosso mapeamento, portanto, foi mais exploratória/descritiva do que exploratória/analítica. Nesse sentido, é importante ressaltar que nosso estudo considerou, unicamente, os títulos das produções bibliográficas. Em outras palavras, nosso objeto de investigação foram as próprias referências bibliográficas.

Tratando-se de um primeiro estudo exploratório dessa natureza, os procedimentos metodológicos que utilizamos foram diversificados, contemplando, por exemplo, consultas randômicas em motores de buscas (como Google e Google Scholar), consultas sistemáticas em plataformas oficiais (plataforma Lattes, domínio público, bancos de teses) e anais de eventos (particularmente na produção respectiva ao campo do Ensino de Matemática).

\section{Resultados: cenário específico do mapeamento das referências bibliográficas acerca do Ensino de Matemática}

Cavalcanti (2015) inventariou nos apêndices A, B, C e D um total de 241 referências que permitiram esboçar o cenário geral da literatura científica brasileira acerca da noção de relação ao saber. Além desse cenário geral, buscou sistematizar, também, o cenário específico da produção científica referente ao contexto do Ensino de Matemática. Assim, delimitou um subconjunto composto por $68(28 \%)$ referências que foram inventariadas nos apêndices $\mathbf{E}(04$ teses de doutorado), F (17 dissertações de mestrado), G (07 artigos em periódicos) e $\mathbf{H}$ (40 artigos em anais de eventos).

No presente artigo, limitamo-nos a analisar 28 referências inventariadas nos apêndices E, F e G de Cavalcanti (2015). Essas referências que foram nosso objeto de análise constam no final do artigo (Anexo A). Os dados das referências do apêndice $\mathbf{H}$, respectivos às comunicações em anais de eventos, ainda não foram analisados. Sendo assim, a análise foi organizada em três (3) categorias identificadas como territórios de produção científica, cada qual respectiva a um tipo de produção (teses, dissertações e artigos publicados em periódicos).

\section{Território 1: teses}

Das 17 teses repertoriadas no apêndice A, 23\%, isto é, quatro (4) destas 17 teses são correspondentes ao contexto do Ensino de Matemática. As referências destas teses estão organizadas no apêndice E. O Quadro 1 apresenta os dados dos autores, ano e respectivos orientadores das teses.

Como podemos observar, três (3) das quatro (4) teses foram defendidas na década de 2010. Apenas uma (1), a tese de Marcos Rogério Neves, foi defendida anteriormente, em 2007. Por conseguinte, provavelmente, essa pode ter sido a primeira tese defendida no campo do Ensino de Matemática. 
Quadro 1. Síntese das teses por autor, ano e orientador(a)

\begin{tabular}{|l|l|c|l|}
\hline $\mathbf{N}^{\mathbf{o}}$ & \multicolumn{1}{|c|}{ Autor } & \multicolumn{1}{c|}{ Ano } & \multicolumn{1}{c|}{ Orientador(a) } \\
\hline 1. & Marcos Rogério Neves & 2007 & Alice H. Campos Pierson \\
\hline 2. & Vanessa Largo & 2012 & Sergio de Mello Arruda \\
\hline 3. & Vinícius Pazuch & 2014 & Maurício Rosa \\
\hline 4. & Itamar Miranda da Silva & 2014 & Tadeu Oliver Gonçalves \\
\hline
\end{tabular}

Fonte: Cavalcanti (2015).

\section{Distribuições por regiões, universidades e programas de pós-graduação}

As quatro teses foram desenvolvidas em quatro instituições distintas, sendo duas na região Sul: Universidade Luterana do Brasil (ULBRA) e Universidade Estadual de Londrina (UEL); uma na região Sudeste: Universidade Federal de São Carlos (UFSCAR); e uma na região norte, na Universidade Federal do Pará (UFPA). A tese de Marcos Rogério Neves foi desenvolvida no doutorado do Programa de Pós-graduação em Educação da UFSCAR. A tese de Vanessa Largo foi desenvolvida no doutorado do Programa de Pós-graduação em Ensino de Ciências e Educação Matemática da UEL. Já a tese de Vinícius Pazuch foi desenvolvida no doutorado do Programa de Pós-graduação em Ensino de Ciências e Matemática da ULBRA e a de Itamar Miranda da Silva, por sua vez, no doutorado do Programa de Pós-graduação em Educação em Ciências e Matemática da UFPA. Como acabamos de relatar, apenas a tese de Marcos Rogério Neves foi desenvolvida no doutorado em Educação (no geral). A demais, foram desenvolvidas em programas de Ensino/Educação em Ciências e Matemáticas.

\section{Território 2: dissertações}

Na lista do apêndice B de Cavalcanti (2015) foram inventariadas 55 referências respectivas a dissertações de mestrado contendo, no título, alusão à noção de relação ao saber. Destas $55,31 \%$, isto é, 17 das dissertações, foram identificadas como relacionadas ao contexto do Ensino de Matemática. As referências destas dissertações estão organizadas no apêndice $\mathbf{F}$ de Cavalcanti (2015). O Quadro 2 apresenta os dados dos autores, ano e respectivos orientadores.

A primeira dissertação que identificamos como vinculada ao contexto do Ensino de Matemática, na qual o título faz menção à noção de relação ao saber, foi defendida por Ronaldo Nogueira Rodrigues e orientada por Anna Franchi. Ressaltamos que essa dissertação, pelo que inventariamos em nosso estudo mais geral, também foi identificada como a primeira dissertação produzida no Brasil sobre o tema relação ao saber.

Como podemos observar no quadro 2, no período de 2001 a 2015, apenas nos anos 2004, 2006, 2008, e 2013 não foram identificadas dissertações contendo, no título, menção à noção de relação ao saber. Por outro lado, nos anos de 2003, 2009, 2010, 2011, 2012 e 2015 foram identificadas duas dissertações em cada ano.

As 17 dissertações identificadas foram orientadas por 13 professores. Sendo assim, ressaltamos que três desses professores orientaram duas dissertações. O professor Carlos Roberto Vianna orientou Pires (2003) e Manosso (2012). A professora Márcia Maria Fusaro Pinto, por sua vez, orientou Capuchinho (2002) e Melo (2003). Já o professor Sergio de Mello Arruda orientou Guizelini (2005) e Antunes (2007). 
Quadro 2. Dissertações por autor, ano e orientador(a)

\begin{tabular}{|l|l|l|l|}
\hline $\mathbf{N}^{\mathbf{0}}$ & \multicolumn{1}{|c|}{ Autor } & Ano & \multicolumn{1}{|c|}{ Orientador(a) } \\
\hline 1. & Ronaldo Nogueira Rodrigues & 2001 & Anna Franchi \\
\hline 2. & Denise da Silva Ribas Capuchinho & 2002 & Márcia Maria Fusaro Pinto \\
\hline 3. & Silvana Martins Melo & 2003 & Marcia Maria Fusaro Pinto \\
\hline 4. & Magna Natália Marin Pires & 2003 & Carlos Roberto Vianna \\
\hline 5. & Alessandra Guizelini & 2005 & Sergio de Mello Arruda \\
\hline 6. & Francieli Cristina A.Antunes & 2007 & Sergio de Mello Arruda \\
\hline 7. & Simone A. Castro Kiefer Oliveira & 2009 & Plinio Cavalcanti Moreira \\
\hline 8. & Denize da Silva Souza & 2009 & Bernard Charlot \\
\hline 9. & Carlos Alberto de Souza Cabello & 2010 & Tânia Maria Mendonça Campos \\
\hline 10. & Vilma Conceição da Silva & 2010 & Francisco de Assis Moura \\
\hline 11. & Carla Cristina Pompeu & 2011 & Vinício de Macedo Santos \\
\hline 12. & Cláudia Patrícia Silvério da Silva & 2011 & Claudia Roberta de Araújo Gomes \\
\hline 13. & Jamille Mineo C. de Magalhães & 2012 & Jutta Cornelia Reuwsaat Justo \\
\hline 14. & Marcia Viviane Barbetta Manosso & 2012 & Carlos Roberto Vianna. \\
\hline 15. & Viviane Andrade de Oliveira Dantas & 2014 & Ana Maria Freitas Teixeira \\
\hline 16. & Hérica dos Santos Matos & 2015 & Veleida Anahi da Silva \\
\hline 17. & Juliana Pires da Silva & 2015 & Méricles Thadeu Moretti \\
\hline
\end{tabular}

Fonte: Cavalcanti (2015).

\section{Distribuições por regiões, universidades e programas de pós-graduação}

Analisando as 17 dissertações de mestrado inventariadas como relacionadas ao Ensino de Matemática, identificamos que sete foram produzidas em universidades da região Sudeste; seis em universidades da região Sul e quatro em universidades da região Nordeste. Dessa maneira, conforme os dados de nossa investigação, ressaltamos que nas regiões Norte e Centro-Oeste não foram identificadas dissertações respectivas ao contexto do Ensino de Matemática contendo, no título, menção à noção de relação ao saber.

Assim, na região Sudeste, três das sete dissertações identificadas, no apêndice $\mathbf{F}$ de Cavalcanti (2015) foram desenvolvidas na Universidade Federal de Minas Gerais (UFMG) - Capuchinho (2002), Melo (2003) e Oliveira (2009). As outras quatro dissertações da região Sudeste foram identificadas nas seguintes instituições: Pontifícia Universidade Católica de São Paulo (PUCSP) - Rodrigues (2001) -; Universidade Bandeirantes de São Paulo (UNIBAN) - Cabello (2010) -; Universidade Federal de Ouro Preto (UFOP) - Conceição da Silva (2010) -; Universidade de São Paulo (USP) - Pompeu (2011). Na região Sul, das seis dissertações identificadas, duas foram desenvolvidas na Universidade Federal do Paraná (UFPR) - Pires (2003) e Manosso (2012) -; duas na UEL - Guizelini (2005) e Antunes (2007) -; uma na ULBRA - Magalhães (2012) - e uma na Universidade Federal de Santa Catarina (UFSC) - Pires da Silva (2015). Na região Nordeste, identificamos três dissertações na Universidade Federal de Sergipe - Souza (2009), Dantas (2014) e Matos (2015) -, e uma na Universidade Federal Rural de Pernambuco (UFRPE) - Silvério da Silva (2011).

Analisando a distribuição das dissertações por programas de Pós-graduação, concluímos que todas foram desenvolvidas em cursos de mestrados em Educação (no geral) ou em Educação/Ensino de Ciências e/ou Matemática, Científica e Tecnológica. De maneira mais 
específica, foram seis dissertações em mestrados em Educação (CAPUCHINHO, 2002; MELO, 2003; OLIVEIRA, 2009; PIRES, 2003; POMPEU, 2011; SOUZA, 2009); três em mestrados em Educação Matemática (CABELLO, 2010; RODRIGUES, 2001; SILVA, 2010); sete em mestrados em Educação/Ensino de Ciências e/ Matemática (ANTUNES, 2007; DANTAS, 2014; GUIZELINI, 2005; MAGALHÃES, 2012; MANOSSO, 2012; MATOS, 2015; SILVA, 2011); uma em mestrado em Educação Científica e Tecnológica (SILVA, 2015).

\section{Território 3: Periódicos nacionais}

Das 41 referências bibliográficas respectivas a artigos publicados em periódicos nacionais que foram repertoriadas no apêndice $\mathbf{C}$ de Cavalcanti (2015), identificamos que 17\%, isto é, sete artigos estão relacionados ao contexto do Ensino de Matemática. Essas respectivas referências estão disponíveis no apêndice $\mathbf{G}$ de Cavalcanti (2015). A partir dessa lista particular, examinamos as informações de modo a organizá-las evidenciando os autores e os periódicos nos quais os artigos foram publicados.

\section{Dos autores}

Averiguando a questão da autoria, constatamos que três dos sete artigos são assinados por apenas um autor: Douady ${ }^{9}$ (1994); Pompeu (2013) e Silva (2008); quatro por um autor e coautor: Oliveira e Moreira (2010); Pinto e Melo (2003); Santos e Curi (2010); Silva e Moura (2011).

Nesse sentido, temos 11 autores associados aos sete artigos. É interessante pontuar a correlação desses trabalhos com autores e orientadores de dissertações. Desse modo, destacamos que os artigos de Oliveira e Moreira (2010), Silva e Moura (2011) e Pinto e Melo (2003) parecem ter relação com as dissertações desenvolvidas no mestrado, uma vez que observamos que Simone Amorim Castro Kiefer Oliveira foi orientada por Plínio Cavalcanti Moreira, Vilma Conceição da Silva foi orientada por Francisco de Assis Moura e Silvana Martins Melo foi orientada por Marcia Maria Fusaro Pinto.

\section{Dos periódicos}

Consideramos importante situar onde os artigos foram publicados. Organizamos, portanto, no Quadro 3, as informações sobre os periódicos identificados e as respectivas publicações.

Conforme pode ser visualizado no Quadro 3, cada artigo foi publicado em um periódico diferente. Explorando os sete periódicos, apuramos que três são pertencentes ao campo da Educação (Em Aberto, Revista Trabalho e Educação, Revista Brasileira de Educação), dois ao campo da Educação Matemática (Zetetiké, Bolema), um ao campo do Ensino de Ciências e Matemática (REnCiMA) e um ao campo da Psicanálise (Estilos da Clínica).

\footnotetext{
${ }^{9}$ Esse artigo corresponde a uma tradução. No entanto, como foi publicado em periódico científico nacional e assim está disponível como parte da literatura científica sobre a noção de relação ao saber no contexto do Ensino de Matemática, optamos por utilizá-lo em nosso mapeamento. O artigo original é: DOUADY, R. Ingénierie didactique et évolution du rapport au savoir: une chronique en calcul mental, un projet en algèbre à l'articulation collège-seconde. Repères IREM, Paris, n. 15, avr. 1994. Disponível em: <http:/ /www.cndp.fr/entrepot/ fileadmin/docs/education_prioritaire/Maths_et_ZEP/reperes15rd.pdf>. Acesso em: 15 out. 2018.
} 
Quadro 3. Periódicos, ano e artigo publicado

\begin{tabular}{|l|c|l|}
\hline \multicolumn{1}{|c|}{ Título do Periódico } & Ano & \multicolumn{1}{c|}{ Artigos publicados } \\
\hline Em Aberto & 1994 & Douady (1994) \\
\hline Revista Trabalho e Educação & 2003 & Pinto; Melo (2003) \\
\hline Revista Brasileira de Educação & 2008 & Silva (2008) \\
\hline Zetetiké & 2010 & Oliveira; Moreira (2010) \\
\hline REnCiMa & 2010 & Santos; Curi (2010) \\
\hline Estilos da Clínica & 2012 & Silva; Moura (2011) \\
\hline Bolema & 2013 & Pompeu (2013) \\
\hline
\end{tabular}

Fonte: Cavalcanti (2015).

\section{Considerações gerais e síntese}

O subconjunto de 68 referências, que constituiu o inventário da produção científica nacional da noção de relação ao saber vinculada ao contexto do Ensino de Matemática, corresponde a 28\% do total de 241 referências repertoriadas em Cavalcanti (2015). Em nossa avaliação, trata-se de um subconjunto significativo. Nessa perspectiva, consideramos que essa representatividade nos permite sugerir, por um lado, que o Ensino de Matemática tem sido um contexto importante na produção científica brasileira referente à noção de relação ao saber e, por outro lado, essa noção tem despertado interesse em pesquisas acerca do Ensino de Matemática.

Para o presente artigo, limitamo-nos a analisar 28 referências (respectivas aos territórios teses, dissertações e artigos publicados em periódicos nacionais). Apesar disso, ponderamos que foi um recorte relevante do subconjunto de referências atinentes ao contexto do Ensino de Matemática, em razão do maior impacto desses tipos (territórios) de produção científica. Levando isso em conta, acreditamos que foi possível apresentar um esboço inicial de um mapa do cenário específico da produção científica correspondente à utilização da noção de relação ao saber nesse contexto específico. Nesse mapa, situamos quando a noção começa a ser utilizada; quantos trabalhos foram desenvolvidos ao longo das últimas décadas; quem utilizou essa noção; onde os estudos foram desenvolvidos e/ou publicados.

Como mapa, esperamos que aqueles que tiverem interesse em utilizar a noção de relação ao saber em estudos vinculados ao campo do Ensino da Matemática possam ter um instrumento de orientação para situar sua investigação na rede das pesquisas já desenvolvidas, permitindo que a produção científica se amplie não apenas em número, mas na coerência e interlocução entre o que se vai produzir, em termos de pesquisa, e o que já foi produzido. Essa tem sido, por exemplo, a postura que temos desenvolvido em nosso Núcleo de Pesquisa da Relação ao Saber (NUPERES) e nas orientações de dissertações nos programas nos quais estamos vinculados.

Por fim, considerando que esse mapeamento foi horizontal (quando, quantos, quem, onde), portanto, de natureza mais descritiva, uma das possibilidades de pesquisa que se abre é o mapeamento vertical como revisão sistemática, cuja natureza mais analítica direcionaria a investigação para "quais avanços que foram conseguidos" e "quais problemas estão em aberto para serem levados adiante". 
A utilização da noção de relação ao saber ...

\section{Referências}

AULAGNIER, P. Le “désir de savoir" dans ses rapports à la transgression. In: BOUHSIRA, J. et al. Transgression. Paris: Presses Universitaires de France, 2009. p. 31-48.

BAUTIER, E.; CHARLOT, B.; ROCHEX, J.-Y. École et savoir dans les banlieues... et ailleurs. Paris: Armand Colin, 1992.

BEILLEROT, J. Le rapport au savoir: une notion em formation. In: BEILLEROT, J. (Coord.). Savoir et rapport au savoir: élaborations théoriques et cliniques. Paris: Éd. Universitaires, 1989. p. 165-202.

BEILLEROT, J. Savoir et rapport au savoir: disposition intime et grammaire sociale. 1987. Thèse (Doctorat d'État) - Université Paris Descartes, Paris, 1987.

BEILLEROT, J. ; BLANCHARD-LAVILLE, C.; MOSCONI, N. Pour une clinique du rapport au savoir. Paris: L'Harmattan, 1996.

BIEMBENGUT, M. S. Mapeamento como princípio metodológico para a pesquisa educacional. In: MACHADO, N. J.; CUNHA, M. O. Linguagem, conhecimento, ação: ensaios de epistemologia e didática. São Paulo: Escrituras, 2003. p. 1-11.

BIEMBENGUT, M. S. Mapeamento na pesquisa educacional. São Paulo: Ciência Moderna, 2008.

BOURDIEU, P.; PASSERON, J-C. La reproduction: éléments pour une théorie du système d'enseignement. Paris: Minuit, 1970.

CAVALCANTI, J. D. B. A noção de relação ao saber: história e epistemologia, panorama do contexto francófono e mapeamento de sua utilização na literatura científica brasileira. Tese (Doutorado em Ensino de Ciências e Matemática) - Departamento de Educação, Universidade Federal Rural de Pernambuco, Recife, 2015. Disponível em: <http://www. tede2.ufrpe.br:8080/tede2/handle/tede2/7458>. Acesso em: 10 out. 2018.

CHARLOT, B. Da relação com o saber: elementos para uma teoria. Porto Alegre: ArtMed, 2000 .

CHEVALLARD, Y. Approche anthropologique du rapport au savoir et didactique des mathématiques. In: MAURY, S.; CAILLOT, M. (Éd.). Rapport au savoir et didactiques. Paris: Fabert, 2003. p. 81-104. Disponível em: <http://yves.chevallard.free.fr/spip/spip/ IMG/pdf/Approche_anthropologique_rapport_au_savoir.pdf>. Acesso em: 10 out. 2018.

CHEVALLARD, Y. Pour une approche anthropologique du rapport au savoir. Dialogue, Toulouse, n. 155, p. 1-11, janv. 2015. Disponível em: <http://www.gfen.asso.fr/images/ documents/publications/dialogue/dial155_enligne_anthropo_rap_savoir_chevallard.pdf $>$. Acesso em: 10 out. 2018.

HATCHUEL, F. Savoir, apprendre, transmettre: une approche psychanalytique du rapport au savoir. Paris: La Découverte, 2005.

LACAN, J. Ecrits. Paris: Le Seuil, 1966. 
Cavalcanti, J. D. B.; Lima, A. P. A. B.

LAOT, F. F. Formateurs d'adultes et diffusion de la notion de rapport au savoir: approche socio-historique. In: HOFSTETTER, R. et al. Savoirs en(trans)formation. Louvain-laNeuve: De Boeck Supérieur, 2009. p. 163-183.

MOSCONI, N. Rapport au savoir: approche sócio-clinique. In: SÉMINAIRE RAPPORT AUX SAVOIRS, 2008, Dijon, 2008. Compte-rendu... Dijon: IUFM de Bourgogne, 2008. 
ANEXO A. Produção científica mapeada

\section{Teses}

LARGO, V. O PIBID e as relações de saber na formação inicial de professores de matemática. 2013. Tese (Doutorado em Ensino de Ciências e Educação Matemática) - Universidade Estadual de Londrina, Londrina, 2013. [Orientador: Sergio de Mello Arruda].

NEVES, M. R. O professor de matemática e seus saberes e suas necessidades em relação à sua disciplina. 2007 Tese (Doutorado em Educação) - Universidade Federal de São Carlos, São Carlos, 2007. [Orientadora: Alice H. Campos Pierson]. Disponível em: <https://repositorio.ufscar.br/handle/ ufscar/2204>. Acesso em: 10 out. 2018.

PAZUCH, V. Cyberformação semipresencial: a relação com o saber de professores que ensinam matemática. 2014. Tese (Doutorado em Ensino de Ciências e Matemática) - Universidade Luterana do Brasil, Canoas, 2014. [Orientador: Maurício Rosa].

SILVA, I. M. A relação do professor com o saber matemático e os conhecimentos mobilizados em sua prática. 2014. Tese (Doutorado em Educação em Ciências e Matemáticas) - Instituto de Educação Matemática e Científica, Universidade Federal do Pará, Belém, 2014. [Orientador: Tadeu Oliver Gonçalves]. Disponível em: <http://repositorio.ufpa.br/jspui/handle/2011/8506>. Acesso em: 10 out. 2018.

\section{Dissertações}

ANTUNES, F. C. A. A relação com o saber e o estágio supervisionado em matemática. 2007. Dissertação (Mestrado em Ensino de Ciências e Educação Matemática) - Universidade Estadual de Londrin, Londrina, 2007. [Orientador: Sergio de Mello Arruda].

CABELLO, C. A. S. Relações institucionais para o ensino da noção de juros na transição ensino médio e ensino superior. 2010. Dissertação (Mestrado em Educação Matemática) Universidade Bandeirante de São Paulo, São Paulo, 2010. [Orientadora: Tânia Maria Mendonça Campos, Coorientadora: Marlene Alves Dias].

CAPUCHINHO, D. S. R. Fatores que influenciam a relação dos alunos com a matemática. 2002. Dissertação (Mestrado em Educação) - Universidade Federal de Minas Gerais, Belo Horizonte, 2002. [Orientadora: Márcia Maria Fusaro Pinto].

DANTAS, V. A. O. A relação com o saber matemático de adolescentes em cumprimento de medida socioeducativa: sentidos e significados em um espaço privado de liberdade. 2014. (Mestrado em Ensino de Ciências Naturais e Matemática) - Universidade Federal de Sergipe, Aracajú, 2014. [Orientadora: Ana Maria Freitas Teixeira].

GUIZELINI, A. Um estudo sobre a relação com o saber e o gostar de matemática, química e biologia. 2005. Dissertação (Mestrado em Ensino de Ciências e Educação Matemática) Universidade Estadual de Londrina, Londrina, 2005. [Orientador: Sergio de Mello Arruda].

MAGALHÃES, J. M. C. Ressignificação de concepções de professores polivalentes sobre sua relação com a matemática e o uso de jogos matemáticos. 2012. Dissertação (Mestrado em Ensino de Ciências e Matemática) - Universidade Luterana do Brasil, Canoas, 2012. [Orientadora: Jutta Cornelia Reuwsaat Justo]. Disponível em: < http://www.ppgecim.ulbra.br/teses/index.php/ ppgecim/article/download/163/156>. Acesso em: 10 out. 2018. 
MANOSSO, M. V. B. Relações com o saber: professores de matemática e seus pontos de vista sobre a formação continuada no estado do Paraná. 2012. Dissertação (Mestrado em Educação em Ciências e Educação Matemática) - Universidade Federal do Paraná, Curitiba, 2012. [Orientador: Carlos Roberto Vianna]. Disponível em: <http://www.exatas.ufpr.br/portal/ppgecm/wp-content/uploads/ sites/27/2016/03/013_MarciaVivianeBarbettaManosso.pdf>. Acesso em: 10 out. 2018.

MATOS, H. S. Relação com o saber em aulas para detentos: a matemática como instrumento de liberdade. 2015. Dissertação (Mestrado em Ensino de Ciências Naturais e Matemática) - Universidade Federal de Sergipe. Aracajú, 2015. [Orientadora: Veleida Anahi da Silva].

MELO, S. M. Um estudo das relações dos alunos com os saberes matemáticos escolares. 2003. Dissertação (Mestrado em Educação) - Universidade Federal de Minas Gerais, Belo Horizonte, 2003. [Orientadora: Marcia Maria Fusaro Pinto].

OLIVEIRA, S. A. C. K. Relação com o saber matemático de alunos em risco de fracasso escolar. 2009. Dissertação (Mestrado em Educação) - Faculdade de Educação, Universidade Federal de Minas Gerais, Belo Horizonte, 2009. [Orientador: Plinio Cavalcanti Moreira].

PIRES, M. N. M. Relação com o saber: alunos de um curso de licenciatura em matemática. 2003. Dissertação (Mestrado em Educação) - Universidade Federal do Paraná, Curitiba, 2003. [Orientador: Carlos Roberto Vianna].

POMPEU, C. C. A experiência escolar de alunos jovens e adultos e sua relação com a Matemática. 2011. Dissertação (Mestrado em Educação) - Faculdade de Educação, Universidade de São Paulo, São Paulo, 2011. [Orientador: Vinício de Macedo Santos]. Disponível em: < http://www. teses.usp.br/teses/disponiveis/48/48134/tde-04072011-152859/pt-br.php>. Acesso em: 10 out. 2018.

RODRIGUES, R. N. Relações com o saber: um estudo sobre o sentido da matemática em uma escola pública. 2001. 167 f. Dissertação (Mestrado em Educação Matemática) - Centro das Ciências Exatas e Tecnologias, Pontifícia Universidade Católica de São Paulo, São Paulo, 2001. [Orientadora: Anna Franchi]. Disponível em: <https://tede2.pucsp.br/handle/handle/11185>. Acesso em: 10 out. 2018.

SILVA, C. P. S. Desempenho escolar em função do gênero na matemática: um estudo sobre as representações da relação com o saber. 2011. Dissertação (Mestrado em Ensino das Ciências) Departamento de Educação, Universidade Federal Rural de Pernambuco, Recife, 2011. [Orientadora: Claudia Roberta de Araújo Gomes].

SILVA, J. A relação com saber: alunos de engenharia e a primeira disciplina de cálculo. 2015. Dissertação (Mestrado em Educação Científica e Tecnológica) - Universidade Federal de Santa Catarina, Florianópolis, 2015. [Orientador: Méricles Thadeu Moretti].

SILVA, V. C. A relação de estudantes do ensino médio de uma escola pública de Mariana - MG com o saber matemático e suas implicações no desempenho escolar em matemática. 2010. Dissertação (Mestrado em Educação Matemática) - Universidade Federal de Ouro Preto, Ouro Preto, 2010. [Orientador: Francisco de Assis Moura].

SOUZA, D. S. A relação com o saber: professores de matemática e práticas educativas no ensino médio. 2009. Dissertação (Mestrado em Educação) - Núcleo de Pós-graduação em Educação, Universidade Federal de Sergipe, Aracajú, 2009. [Orientador: Bernard Jean Jacques Charlot]. 


\section{Artigos em periódicos}

DOUADY, R. Evolução da relação com o saber em matemática na escola primária: uma crônica sobre calculo mental. Em Aberto, Brasília, v. 14, n. 62, p. 33-42, 1994. Disponível em: < http://emaberto. inep.gov.br/index.php/emaberto/article/view/1962/1931>. Acesso em: 10 out. 2018.

OLIVEIRA, S. A. C. K.; MOREIRA, P. C. Relação com o saber matemático de alunos em risco de fracasso escolar. Zetetiké, Campinas, v. 18, n. 33, p. 239-266, 2010. Disponível em: <http://ojs. fe.unicamp.br/ged/zetetike/article/view/2817/2474>. Acesso em: 10 out. 2018.

PINTO, M. M. F.; MELO, S. M. A relação com o saber na sala de aula de matemática. Revista Trabalho e Educação, Belo Horizonte, v. 12, n. 1, p. 127-135, 2003. Disponível em: < https://seer. ufmg.br/index.php/trabedu/article/view/7370>. Acesso em: 10 out. 2018.

POMPEU, C. C. Aula de matemática: as relações entre o sujeito e o conhecimento matemático. Bolema, Rio Claro, v. 27, n. 45, p. 303-321, 2013. Disponível em: <https://doi. org/10.1590/S0103-636X2013000100015>. Acesso em: 10 out. 2018.

SANTOS, C. A. B.; CURI, E. Proposta curricular de matemática: uma análise da relação institucional esperada para as noções de área e perímetro. REnCiMa, v. 1, n. 1, p. 27-33, 2010. Disponível em: $<$ http://revistapos.cruzeirodosul.edu.br/index.php/rencima/article/viewFile/5/5>. Acesso em: 10 out. 2018.

SILVA, V. A. Relação com o saber na aprendizagem matemática: uma contribuição para a reflexão didática sobre as práticas educativas. Revista Brasileira de Educação, Rio de Janeiro, v. 13, n. 37, p. 150-190, 2008. Disponível em: <http://www.scielo.br/pdf/rbedu/v13n37/13.pdf>. Acesso em: 10 out. 2018.

SILVA, V. C.; MOURA, F. A. A relação com o saber e suas implicações no desempenho escolar em matemática. Estilos da Clínica, São Paulo, v. 16, n. 2, p. 442-459, 2011. Disponível em: < http:/ / www.revistas.usp.br/estic/article/view/46128/49753>. Acesso em: 10 out. 2018.

Artigo recebido em 16/02/2018. Aceito em 31/05/2018.

Universidade Federal de Pernambuco, Centro Acadêmico do Agreste, Sala 21-CAA, Rodovia BR-104, Km 59, s/n, Nova Caruaru, PE, 55002-970, Brasil. 
